\title{
Juana Borrero oder die Liebe als Rebellion
}

Juana Borrero ist ein Wunderkind der Literatur und der Malerei. Und genau dies, ihr Ruf als Wunderkind, dürfte wohl zusammen mit der Kürze ihres Lebens dazu geführt haben, dass sie in einer von Männern beherrschten kubanischen Literaturszene als Lyrikerin niemals gänzlich ernstgenommen wurde. Doch beschäftigen wir uns zunächst mit einigen wenigen biographischen Daten, um die materielle Existenz der einzigen kubanischen Modernistin genauer zu fassen!

Juana wurde am 18. Mai 1877 in Havanna geboren und starb am 9. März 1896 in Cayo Hueso, also Key West in Florida. Juana war die Tochter des kubanischen Arztes und Lyrikers Esteban Borrero Echeverría und entstammte einer wahren Dichterdynastie. Sie wurde wie ihre Schwestern in einem erlesenen Ambiente von Kunst und Literatur erzogen. Schon als junges Mädchen erhielt sie privaten Zeichenunterricht von Dolores Desvernine und stellte rasch ihr immenses Talent unter Beweis, so dass man sie 1886 in die Academia de Bellas Artes de San Alejandro aufnahm. Später wurde sie zur Schülerin des Malers Armando Menocal und reihte sich damit ein in eine wichtige Traditionslinie der kubanischen Malerei. Kommen Sie heute nach La Habana, so können sie im Museo de Bellas Artes einige wenige Arbeiten dieser herausragenden Malerin bewundern. Aber die Mal- und Zeichenkunst bildete nur eine Seite ihrer vielfältigen Talente.

Denn Juana Borrero war vor allem auch Lyrikerin! Ihre große Liebe galt dem kubanischen Modernisten Julián del Casal, den sie zutiefst bewunderte und in den sie sich als noch jugendliches Mädchen verliebte. Ihre frühen Gedichte erschienen in den großen kubanischen Zeitschriften der Zeit, so dass ihr bald schon der Ruf als Wunderkind der kubanischen Lyrik vorauseilte. 1892 reiste sie mit ihrem Vater nach New York und lernte dort, in seinem Exil, den großen kubanischen Lyriker, Essayisten und Revolutionsführer José Martí kennen, der ihr zu Ehren in Chickering Hall eine Velada literaria abhielt. ${ }^{1}$ In Washington nahm sie weiter Malunterricht bei Mac Donald, während ihr Vater seine Beziehungen zum kubanischen Exil intensivierte. 1893 kehrte sie nach Kuba zu ihrer Familie zurück, wo ihre Schwestern innerhalb der patriarchalischen Familienstruktur die wichtigsten Ansprech- und Austauschpartnerinnen waren, mit denen sie ihre Geheimnisse zu teilen suchte.

1 Vgl. hierzu auch Ette, Ottmar: Geschlechtermodellierungen - Geschlechtergrenzen. José Martí und Juana Borrero. In: Krüger, Reinhard (Hg.): Grenzgänge, Hybride \& Fusionen. Romanistische Beiträge zu polykulturellen Kommunikationsprozessen. Berlin: Weidler Buchverlag 2008, S. 21-39.

Ә Open Access. (C) 2020 Ottmar Ette, publiziert von De Gruyter. (ल) BY-NC-ND Dieses Werk ist lizenziert unter der Creative Commons Attribution-NonCommercial-NoDerivatives 4.0 International Lizenz. https://doi.org/10.1515/9783110665093-020 


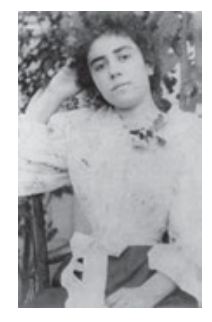

Abb. 99: Juana Borrero (Havanna auf Kuba, 1877 - Key West, Florida, 1896).

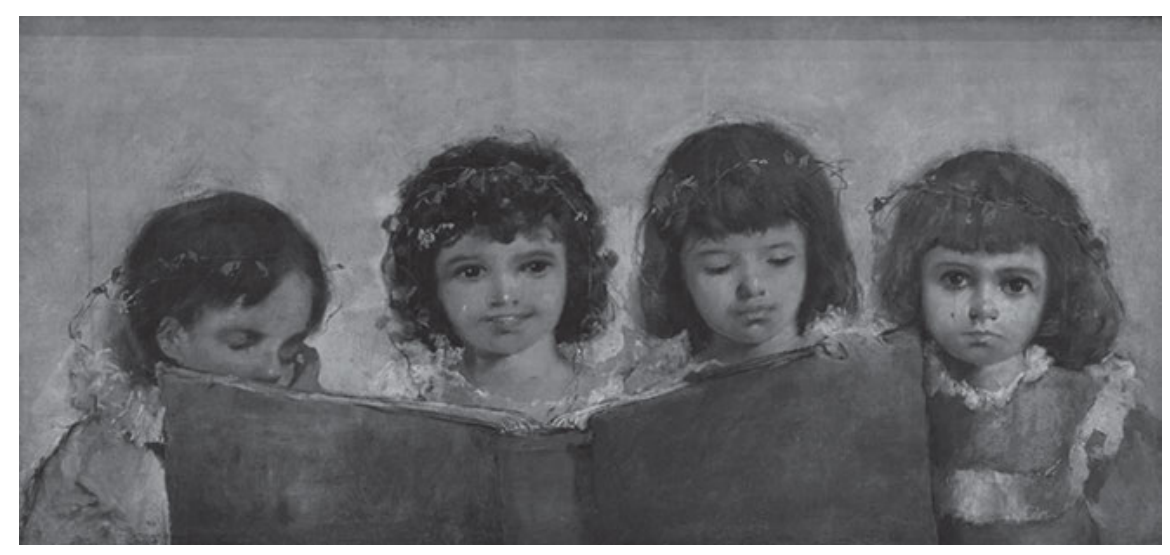

Abb. 100: „Las niñas“, Öl auf Leinwand von Juana Borrero, circa 1895.

$\mathrm{Zu}$ Lebzeiten wurde Juana Borrero in Kuba überwiegend als Teil ihrer Dichterfamilie wahrgenommen. Einige ihrer Gedichte erschienen in der Anthologie Grupo de familia, poesías de los Borrero im Jahr 1895. Sie veröffentlichte ihre Gedichte aber zunehmend auch in La Habana Elegante, El Fígaro sowie in Gris y Azul und machte sich als kubanische Modernistin einen Namen. Über ihre umfangreiche Korrespondenz, darunter ihre berühmten Liebesbriefe, die Cartas de amor, und ihre Gedichte hinaus hinterließ sie zahlreiche Zeichnungen und Gemälde, von denen nicht alle erhalten blieben. 1895 emigrierte sie mit ihrer ganzen Familie ihr Vater hatte sich für den Aufstand gegen die spanische Kolonialherrschaft eingesetzt - krank, geschwächt und widerstrebend nach Key West in Florida, wo sie im Alter von achtzehn Jahren verstarb.

An anderer Stelle habe ich versucht, ihre Malerei- wie ihre Dichtkunst in einer Bild und Text miteinander verbindenden Zusammenschau $\mathrm{zu}$ würdigen. ${ }^{2}$ Eine solche Vision kann ich an dieser Stelle leider nicht einbauen, doch möchte

2 Vgl. Ette, Ottmar: Juana Borrero: convivencia y transvivencia. In: Rodríguez Gutiérrez, Milena (Hg.): Casa en la que nunca he sido extraña. Las poetas hispanoamericanas: identidades, 
ich im Folgenden einige wesentliche Aspekte ihres Künstlerlebens als modernista zumindest ausschnitthaft beleuchten.

Im Februar 1895, zu einem Zeitpunkt, als José Martí vom kubanischen Exil aus intensiv und ruhelos mit den Vorbereitungen ,seines‘ Krieges und der Invasion Kubas beschäftigt war, notierte eine erregte Juana Borrero des Nachts in ihrem Tagebuch:

Ich habe sehr schnell und ohne mich aufzuhalten die Verse von Federico gelesen. Sie faszinieren mich. Aber ich weiß nicht, warum mich Carlos ... mich mit seinem rätselhaften und traurigen Aussehen anzieht. Ich lese noch einmal seine Strophen. Eingeschlossen ... Wird er aufrichtig sein?! Oh mein Gott, so sieht der Mann aus, von dem ich geträumt! Warum hast Du ihn so weit entfernt von mir gestellt? [...]

Nacht.

Es ist halb drei Uhr. Ich habe nicht geschlafen und werde es auch nicht. Ich habe gerade etwas Unerhörtes, Unmögliches, Hochgewagtes gedacht. Hör zu, Carlos! Noch ehe zwei Monate vorüber sind, wirst Du mein sein oder ich tot. ${ }^{3}$

Dies sind wahrlich starke Worte eines jungen Mädchens! Die siebzehnjährige Juana Borrero sollte das sich selbst gegebene Versprechen, die dem Mann in Abwesenheit entgegengeschleuderte Herausforderung einlösen und den Dichter mit dem rätselhaften und traurigen Ausdruck, Carlos Pío Uhrbach, zu dem Ihren machen. Die Tagebucheinträge der in eine Familie von Literaten ${ }^{4}$ geborenen jungen Frau, die bereits mit vier Jahren erste Gedichte schrieb und mit fünf die beeindruckende Zeichnung einer Nelke und einer Rose mit dem Titel „Romeo y Julieta" versah, ${ }^{5}$ hatten ihr Bild des Geliebten allein aus der Literatur bezogen und mit jenem des von ihr erträumten Mannes in Übereinstimmung zu bringen versucht. Und diese gleichsam literarisch gestiftete Liebesbeziehung sollte dramatische Folgen haben.

Als Juana Borrero wenige Monate zuvor den soeben erschienenen Gedichtband Gemelas der beiden kubanischen Lyriker Carlos Pío und Federico Uhrbach erhielt, fragte sie sich bereits vor der Lektüre, die sie sich für die Nacht vornahm: „¿Será un compañero de mis insomnios?““(Wird er ein Gefährte meiner schlaflo-

feminismos, poéticas (Siglos XIX - XXI). New York - Bern - Frankfurt am Main: Peter Lang 2017, S. 268-307.

3 Borrero, Juana: Epistolario De Juana Borrero. Habana Cuba,1877 - Cayo Hueso EEUU,1896. 2 Bde. La Habana: Academia de Ciencias de Cuba 1966, Bd. I, S. 41.

4 Vgl. hierzu Cuza Malé, Belkis: El clavel y la rosa. Biografía de Juana Borrero. Madrid: Ediciones Cultura Hispánica 1984.

5 Diese später berühmt gewordene Zeichnung, die auch der Borrero-Biographie der im Exil lebenden kubanischen Lyrikerin Belkis Cuza Malés den Namen gab, findet sich auf Umschlag und Titelseite der beiden Bände des bereits erwähnten Epistolario. 


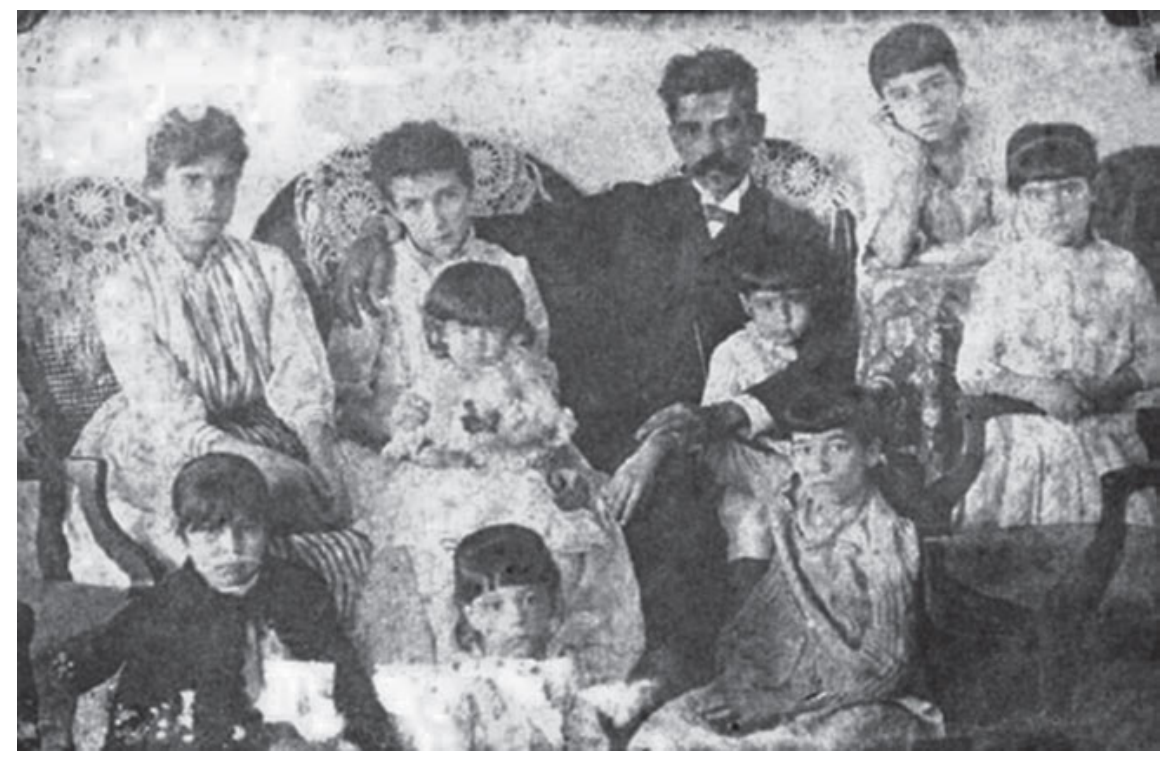

Abb. 101: Fotografie der Familie Borrero.

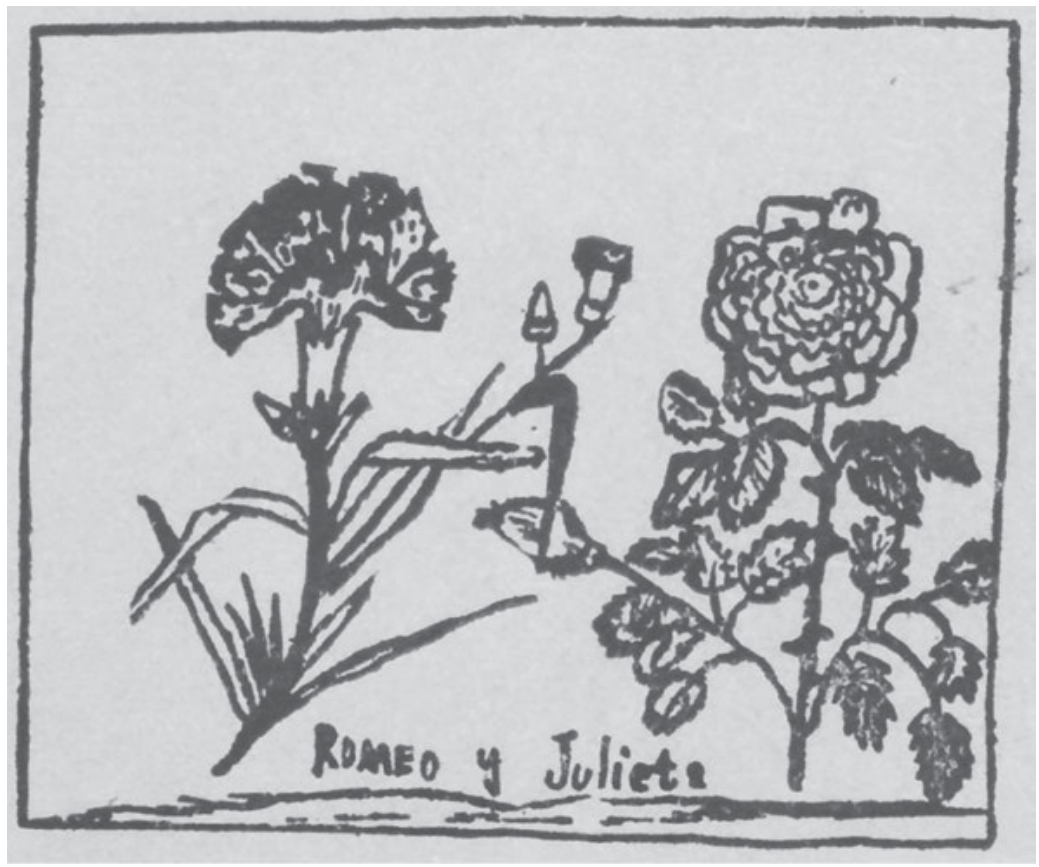

Abb. 102: „El clavel y la rosa“, Zeichnung von Juana Borrero, 1882. 
sen Nächte sein?) ${ }^{6}$ Schien damit zunächst das Buch und die darin versammelten Gedichte gemeint zu sein, so sprang die Sehnsucht nach einem realen männlichen Gefährten ihrer schlaflosen Nächte schnell über auf den älteren der beiden Uhrbach-Brüder, dessen Verse sie ebenfalls faszinierten und unwiderstehlich anzogen. Das rasch sich bei der jungen Malerin durch Lektüre hypotypotisch in ihrer Imagination einstellende Portrait war sicher und unsicher zugleich:

Ich habe mehrere Seiten gelesen, oh Carlos!, und ich kann Dir jubilierend sagen, dass ich keinerlei Enttäuschung fühlte. In diesen Versen ist etwas Originelles, das faszinierend anzieht und anziehend ... fasziniert. Das erste Portrait ...! Es ist ein stolzes Antlitz. Carlos muss wohl bleich sein, ein Kranker. Da ist auf seiner edlen Stirne ein unmerklicher Zug von Überdruss. Vielleicht täusche ich mich - Ich werde heute Nacht nicht weiterlesen ... Ich nehme an, dass ich ganz im ersten Teil des Bandes verweilen werde. Carlos muss wohl leiden ... Aber alles in allem, was kümmert mich das ...? Denn ich werde ihn niemals sehen noch wird er jemals wissen, dass ich jetzt beim Einschlafen seinen Namen murmle ... ${ }^{7}$

Zunächst können wir feststellen, dass der Juana Borrero faszinierende Carlos mit jenen Zeichen ausgestattet ist (oder von der jungen Frau ausgestattet wird), welche präzise dem idealen Menschenbild im europäischen wie amerikanischen Fin de siècle entsprechen. Denn Carlos ist nicht nur bleich, sondern von jenem tedio, von jenem ennui, von jenem Überdruss geprägt, der sich bei den Heldinnen und Helden des Jahrhundertendes auf die gesamte Gesellschaft bezieht. Auch fehlt ihm nicht jene Krankheit, die noch immer das Zeichen eines höheren, eines überlegenen Geistes ist. War das mal du siècle an der Wende zum 19. Jahrhundert die Melancholie, so ist es nun, an der Wende zum 20. Jahrhundert, der durch nichts aufzulösende ennui, der gewaltige Überdruss an der Gesellschaft.

Doch wenden wir uns der Niederschrift dieser Zeilen und jener écriture zu, welche Juana Borrero in ihren Briefen an Carlos Pío Uhrbach entwickelte und an der sie immer weiter feilte! Ist die teilweise auftretende Agrammatikalität die nicht mit einem krankhaften Agrammatismus zu verwechseln ist - Beleg einer raschen Niederschrift oder des Stils dieser Tagebuchaufzeichnung, der in den Bereich des Stream of Consciousness rückt? In jedem Falle können wir festhalten - und dies ist ein recht spannender Fall von gender trouble -: Juana Borreros Männerideal ist idealerweise kein Mann! „Wird er im Übrigen das Ideal des Mannes erfüllen, den ich erträumt habe? Seine Verse versprechen dies, aber ist er vielleicht kein Mann?“8

6 Borrero, Juana: Epistolario, Bd. I, S. 39.

7 Ebda.

8 Ebda., S. 40f: „Además ¿realizará él el ideal del hombre que he soñado? sus rimas lo prometen, pero ¿acaso no es un hombre?“. 
Diese Formel verrät viel über das paradoxe Verhältnis von Leben, Lesen und Lieben, das sich um den imaginierten Mann aufbaut und Juana Borrero bis zu ihrem tragischen Tod im nordamerikanischen Exil im März 1896 nicht mehr loslassen sollte. Blenden wir an dieser Stelle eine ebenso denkwürdige wie in ihrer Absolutheit fragwürdige Behauptung von Julia Kristeva ein, insofern die These der Psychoanalytikerin und Literaturwissenschaftlerin vielleicht zusätzliches Licht auf diese Tagebucheinträge oder Briefe der Juana Borrero zu werfen vermag: „Was heißt lieben für eine Frau, dasselbe wie schreiben“. ${ }^{9}$ Auf die Agrammatikalität auch dieses Satzes sei an dieser Stelle nur hingewiesen.

Fragen wir uns also: Wurde Juana Borrero zu einem simplen Opfer der Lektüre in der Nachfolge von Dantes Paolo und Francesca oder den unsterblich-sterblich Verliebten in Jorge Isaacs' María? Fast will es so scheinen, zumal die Nähe zur romantischen Disposition imaginierter Männlichkeit gerade durch einen Vergleich mit der in Puerto Príncipe, dem heutigen Camagüey - auch Juanas Vater, der Lyriker und Arzt Esteban Borrero stammte von dort - geborenen Gertrudis Gómez de Avellaneda deutlich wird. In ihrer 1839 in Briefen an ihren damaligen Geliebten verfassten Autobiographie skizzierte die in Madrid berühmt gewordene und in Havanna zur Dichterin gekrönte Lyrikerin, der Juanas Großvater, der ebenfalls ins Exil getriebene Dichter Esteban de Jesús Borrero gehuldigt hatte, ihre eigene Kindheit im Alter von dreizehn Jahren:

\begin{abstract}
Später wurde die gemeinsame Lektüre von Romanen, Gedichten und Komödien zu unserer alles beherrschenden Leidenschaft. Mama schimpfte manchmal mit uns, dass wir doch schon so groß seien und so sehr unseren Schmuck vernachlässigten und vor der Gemeinschaft Ausreiss nähmen, als wären wir Wilde. Denn unsere größte Lust war es, im Zimmer mit unseren Büchern eingeschlossen zu sein und unsere Lieblingsromane zu lesen, wobei wir heiße Tränen vergossen angesichts der unglücklichen Episoden unserer imaginären Helden, die wir so sehr liebten. ${ }^{10}$
\end{abstract}

Zweifellos hatte auch Juana Borrero jene Sozialisation als Mädchen und junge Frau erfahren, auf welche die große kubanische Dichterin der Romantik stolz und - wie wir sahen - in Abgrenzung zur Erziehung der Frauen im spanischen Mutter-Land verwies: eben nicht für das gemacht zu sein, was man in Spanien für die „obligación de su sexo“, die Verpflichtungen des Geschlechts, hielt, worunter man Bügeln, Kochen, Sockenstopfen, Bettenmachen und Putzen verstehe. Vielmehr lernten die junge Tula wie auch die junge Juana, sich den Büchern und dem Studium der höheren Dinge zu widmen, weshalb man die gute Gertrudis später in

9 Kristeva, Julia: Histoires d'amour. Paris: Editions Denoël 1983, S. 296: „Qu'est-ce aimer, pour une femme, la même chose qu'écrire.“

10 Gómez de Avellaneda, Gertrudis: Autobiografía, S. 52f. 
Spanien als , la doctora“ verspottete. ${ }^{11}$ Bis in die Liebesmetaphorik hinein verband gewiss vieles die große Vertreterin und Hoffnung des „ersten Modernismo unter uns“12 mit der Lyrikerin des Romanticismo; doch hatten sich trotz aller Kontinuitäten die ästhetischen Parameter zwischen Juana und Tula gewandelt.

Juana Borrero lebte und liebte die Lektüre: Sie war ein Wesen ganz in der Welt der Bücher, welche sie vor der umgebenden Realität beschützten und für sie eine Welt kreierten, in welcher sie ihre Begierden und Bedürfnisse ausleben konnte. An ihrem Lesen richtete sich auch ihr Lieben aus mit einem Anspruch, der im Verhältnis zu der ihr als Frau in einer patriarchalischen Gesellschaft zugebilligten Rolle disproportional war. Denn so, wie sich der von ihr erträumte Mann Carlos Pío Uhrbach zumindest über einen langen Zeitraum bemühte, dem auf ihn projizierten Männerbild eines Nicht-Mannes zu entsprechen, so sah Juana Borrero ihr eigenes Bild und mehr noch ihr Schicksal durch ein Gedicht vorgeformt, das der große Dichter des kubanischen Modernismo, Julián del Casal, unter dem Titel „Virgen triste“ veröffentlicht hatte.

In diesem Gedicht des früh verstorbenen Dichters sah Juana Borrero ihr Bildnis bis hinein in einen frühen Tod literarisch vorgeformt. Die prospektive Dimension von Literatur war für sie gleichsam prophetisch geworden und konnte durch die Lektüre angeeignet werden, ja mehr noch: Sie sah sich selbst als Verkörperung dessen, was sie in diesem Gedicht gelesen hatte. Dessen letzte Strophe wurde ihr zur Weissagung des eigenen frühen Todes - und auch dies war ein Versprechen der Literatur, welches das Leben einlösen sollte:

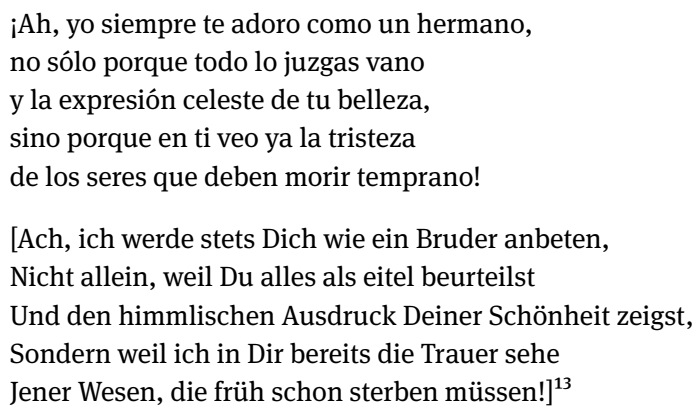

11 Ebda., S. 68.

12 Vitier, Cintio: Las cartas de amor de Juana Borrero. In: Borrero, Juana: Epistolario. Bd. I, S. 31: „primer modernismo entre nosotros“.

13 Casal, Julián: Poesía completa y prosa selecta. Edición a cargo de Álvara Salvador. Madrid: Editorial Verbum 2001, S. 223. 
Die Worte des großen kubanischen Modernisten wogen für Juana Borrero schwer, hatte sich das junge Mädchen doch Hals über Kopf in einen Julián del Casal verliebt, der eine andere Sichtweise der ästhetischen Erneuerung im Schreiben wie im Handeln als José Martí vertrat. Julián del Casal war jener Dichter, der im Gegensatz zu Martí an sein Havanna gebunden blieb und nicht mit einem Exil liebäugelte, in welchem sich die Lebenserfahrung des Dichters der Versos libres mit jener großen Welterfahrung gepaart hatte, die Martí zweifellos in den ästhetischen Leuchtturm seiner Generation verwandelte. Casal blieb in seinen zum Teil scharfen Gesellschaftschroniken stets der kubanischen Hauptstadt verpflichtet und beschäftigte sich intensiv mit deren Gestalten und Figuren.

In einer Juana Borrero gewidmeten Studie, die zunächst in La Habana Literaria erschien und von Casal später in seine berühmten Bustos y Rimas aufgenommen wurde, beschrieb der 1893 kurz vor seinem dreißigsten Geburtstag überraschend verstorbene Dichter, wie er nach einem Besuch im Hause Borrero in Puentes Grandes vor den Toren Havannas erstmals ihr literarisches Portrait auf dem Nachhauseweg („esbocé su retrato por el camino ${ }^{14 “)}$ gleichsam im Gehen entworfen habe. Auch hier lag der Schwerpunkt des Gedichts auf dessen letzter Strophe. Denn hier sehen wir das Bildnis des jungen Mädchens noch einmal aufleuchten:

\author{
¡Doce años! Mas sus facciones \\ Veló ya de honda amargura \\ La tristeza prematura \\ De los grandes corazones. ${ }^{15}$ \\ [Zwölf Jahre! Doch in ihren Zügen \\ Zeigte sich in tiefer Bitterkeit \\ Die allzu verfrühte Traurigkeit \\ Der großen, großen Herzen.]
}

Das Spiel der Literatur mit Leben und Liebe blieb hierbei freilich nicht stehen, ,antwortete‘ doch gleichsam die erste Strophe des von Julián del Casal in seiner Studie als erstes zitierten Gedichts Juana Borreros unter dem Titel „jTodavía!“ auf dieses literarische Portrait:

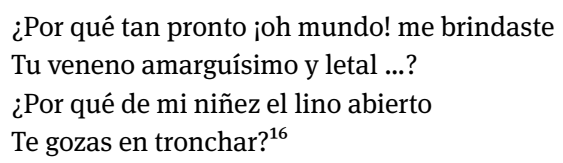

14 Casal, Julián: Juana Borrero. In (ders.): Prosas. Edición del Centenario. Bd. I. La Habana: Consejo Nacional de Cultura 1963, S. 266.

15 Ebda., S. 267.

16 Ebda., S. 268. 
[Warum so früh, oh Welt!, schenktest Du mir ein Dein bitterstes und tödlichstes Gift ...?

Warum gefällt es Dir, von meiner Kindheit noch

Das offene Linnen zu zerreißen?]

Casals Verse blieben für Juana Borrero ein bleibendes Vermächtnis und zugleich eine schwere Hinterlassenschaft, welche der Dichter kaum als eine für die junge Frau so schwierig zu bewältigende erkannt haben dürfte. Diese Echowirkungen der Literatur über das Leben verliehen Juana Borrero von Beginn an eine im doppelten Wortsinn gemeinte literarische Existenz, welche den Interpretationen ihres umfangreichen Schaffens als Künstlerin des Pinsels und der Feder eine grundlegende und bei den bis heute spärlichen Deutungen unverrückte Leitlinie vorgab, die Literatur Juana Borreros mit ihrem Leben kurzzuschließen und geradezu unausweichlich einer ausschließlich autobiographischen Lektüre zuzuführen. ${ }^{17}$

Die Deutung von Juana Borreros Gedichten oder Briefen wurde damit semantisch erheblich, ja bisweilen bis zur Unkenntlichkeit eingeengt. Aus einer derartigen Perspektive wäre auch die Ansicht Cintio Vitiers zu hinterfragen, Juana Borreros Liebesbriefe seien „ihr größtes Werk“, weil sie „ihr größtes Bekenntnis“ darstellten. ${ }^{18}$ Eine derartige Einschätzung läuft Gefahr, die künstlerische und ästhetische Dimension einem bloß autobiographischen Interesse zu opfern. Es gilt aber, die Verschränktheit und Interdependenz von Kunst als Leben und Leben als Kunst im Gesamtwerk der kubanischen Autorin wieder zum Vorschein zu bringen und damit Sorge dafür zu tragen, hinter dem Wunderkind wieder die Künstlerin in ihren literarästhetischen Herausforderungen und Widersprüchen zu sehen. ${ }^{19}$ Es gilt zu den Texten und Werken von Juana Borrero zurückzukehren und sich auf deren Interpretation zu konzentrieren!

Wir sollten uns überdies vor der Fehldeutung hüten, Juana Borrero hätte einem von Männern entworfenen Frauenbild nur nachgeeifert, ihre Subjektwerdung gar an einem romantischen Gemeinplatz ausgerichtet! Irrte sich Julián del Casal auch mit seiner Einschätzung, die junge „Künstlerin mit so brillanten Fähigkeiten“ werde binnen kurzer Zeit „die glühende Markierung der Berühmtheit“ empfangen, so gab er den künftigen Lesern dieser bis heute noch immer kaum bekannten Lyrikerin doch einen wichtigen Hinweis, insofern er abschließend betonte, aller Erfolg werde Juana Borrero niemals von ihrer „,absolutesten

17 Dies gilt für die genannten Studien ebenso wie für neuere Arbeiten wie die mit einem eher dilettantischen Rückgriff auf Lacan operierende Arbeit von Hauser, Rex: Juana Borrero: The Poetics of Despair. In: Letras Femeninas (Ithaca) XVI, 1-2 (primavera - otoño 1990), S. 113-120.

18 Vitier, Cintio: Las cartas de amor de Juana Borrero, S. 25.

19 Vgl. hierzu Ette, Ottmar: Juana Borrero: convivencia y transvivencia, S. 268-307. 


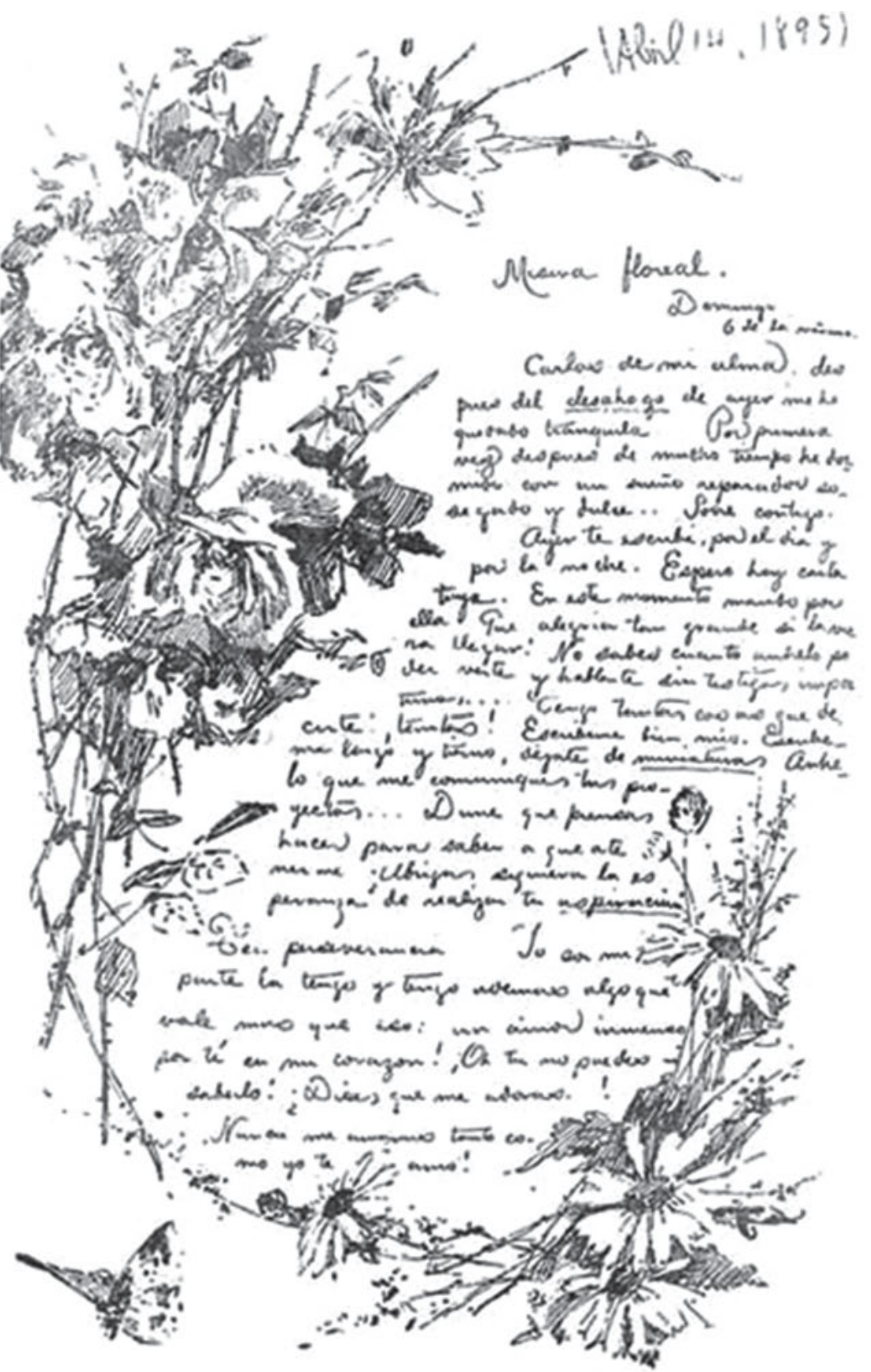

Abb. 103: „Misiva floreal“, Brief von Juana Borrero an Carlos Pío Uhrbach, am 14. April 1895. 
Verachtung“ und der „tiefsten Indifferenz“ gegenüber den „Meinungen der braven Bürger in der Literatur“20 abbringen. Denn Juana Borreros künstlerische wie auch lebensbezogene Überzeugungen waren keinesfalls jene der burgueses de las letras: Sie ging ihren eigenen Weg im Malen, Schreiben und Lesen, im Leben wie in der Liebe.

Denn Juana Borreros Kunstauffassung war ebenso radikal wie absolut. In eine nur bürgerliche Kunstauffassung, nur bürgerliche Lebensauffassung ließ sich ihr Lebensprojekt, das auch zugleich ihr Kunstprojekt war, nicht einzwängen. Die Dreiecksbeziehung von Lesen, Lieben und Leben lässt sich quer durch ihr gesamtes Schaffen verfolgen und findet sich auch personifiziert in Konstellationen, die dem Leben entnommen zu sein scheinen und gleichzeitig (ästhetisiertes) Leben bilden.

So sind Juana Borreros Liebesbriefe an Carlos Pío Uhrbach durchzogen von Anspielungen auf Julián del Casals Leben, von Zitaten seiner Gedichte und ,Erscheinungen“ des Dichters, deren allererste sich nicht zufällig bereits in ihrem ersten Brief an die Brüder Uhrbach findet. Darin lesen wir:

Diese Nacht träumte ich von Casal. Ich sah ihn nicht wie sonst immer ekstatisch und traurig, sondern besorgt und stumm. So blieb er über mehr als zwei Stunden unbeweglich, und als er wieder in der Finsternis verschwand, da schaute er mich mit einem Blick an, in welchem man den Vorwurf erriet ... Warum und wofür sollte ich Schuld tragen? ${ }^{21}$

Julián del Casal ist in den Träumen der jungen Dichterin allgegenwärtig. Sein Bild steht nicht nur für einen jungen, extravaganten und in der Tat nicht selten ekstatischen Mann, sondern für die Kunst und das eigene Leben als Kunstwerk. Zugleich ist sein Bild bei Juana immer wieder mit eigenen Schuldkomplexen belastet, musste sie sich doch vorhalten, bei einer ihrer letzten Begegnungen mit dem Dichter zumindest für Missverständnisse gesorgt $\mathrm{zu}$ haben. Aber man darf in dieser Zurückweisung von Schuld, die letztlich die Obsession der Schuld bekräftigt, auch das Eingeständnis der Malerin und Dichterin erblicken, in der Verwandlung des eigenen Lebens und Liebens in Kunst noch nicht so weit vorgedrungen zu sein wie der stumm und vorwurfsvoll blickende, und zugleich doch früh verstorbene Casal, der am Ende wieder im Dunkel des Traumes, im Dunkel des Raumes, verschwindet.

$\mathrm{Zu}$ Recht hat Cintio Vitier auf die verborgene Präsenz Casals in Gestalt jenes Dolches, den er ihr einst schenkte, in einem weiteren Traum von Juana Borrero aufmerksam gemacht, in dem die Träumende ihren Carlos mit einer anderen Geliebten zu überraschen meinte:

20 Casal, Julián: Juana Borrero, S. 271.

21 Borrero, Juana: Epistolario, Bd. I, S. 42. 


\begin{abstract}
Niemals, solange ich lebe, wird mir jene Frau, jene Unbekannte aus dem Sinn gehen, die nicht existiert und die leise in ein Gespräch vertieft voranschritt. Ich hob die Hand und versenkte den Dolch in ihrem Herzen. Daraufhin geschah etwas, dessen Erinnerung mich noch immer mit Schrecken erfüllt ... Denn jene Frau war ich selbst. In einer Aufwallung von wilder Eifersucht hatte ich mich gerade selbst getötet. Das Leid Deiner Trauer und das unaussprechliche Gefühl, mich für immer tot zu sehen, waren so gewalttätig, dass ich schluchzend aufwachte. Was für ein seltsamer Traum! Ich selbst hatte mich umgebracht und betrachtete zugleich meine Leiche. Ja, in der Tat, die Träume sind bisweilen dunkel und rätselhaft. Mit Tagesanbruch flohen die „Herden von Schmetterlingen des Todes“. Danach habe ich oft an meinen Albtraum gedacht. Mir scheint, ich entdecke in ihm ein geheimes und mysteriöses Symbol. Glaubst Du nicht auch? In jedem Falle war es etwas sehr Trauriges, sehr Trauriges ... Das erste, was ich nach dem Aufwachen tat, war Deine letzten Briefe zu lesen, die ich unter meinem Kopfkissen bewahre. Ihre Lektüre beruhigte mich. Ich küsste sie, vor Zärtlichkeit weinend, und ich presste sie erschreckt an mein Herz. Sie haben mich ein weiteres Mal getröstet. Bringen sie denn nicht Deine Seele zu mir? Ach! Könntest Du in die ganze Tiefe meiner Leidenschaft eindringen! Ich leide, weil ich mir vorstelle, dass ich die Bedürfnisse Deiner Seele nicht befriedigen kann ... Andere Male glaube ich, Dein Ideal zu sein und dann bin ich glücklich, oh ja, sehr glücklich! ... In diesem Augenblick bin ich glückselig. Morgen sehe ich Dich. Oh Hoffnung! Du bist die große Trösterin! ${ }^{22}$
\end{abstract}

Der Abschluss dieser dichten und ergreifenden Passage ist durchaus ein wenig zweideutig, denn der Mann wird hier - syntaktisch parallel zur Hoffnung - gleichsam in ein weibliches, vielleicht auch geschlechtloses Wesen verwandelt. Dies werden wir später noch etwas näher betrachten können. Zunächst aufschlussreicher sind die großen Zusammenhänge im Aufbau der Liebesbriefe Juana Borreros an Carlos Pío Uhrbach. Denn in dieser Passage beginnt, was man in Anschluss an Roland Barthes eine Serie der ,Figuren der Liebe‘ nennen könnte. Es handelt sich zugleich um eine Art künstlerischer Figurenfolge, wie sie Giacomo Casanova in seinen Schriften auf so ganz andere Weise und mit immer neuen Liebesobjekten durchspielte. Genau dies vollführt Juana Borrero an nur einem einzigen, ihrem literarisierten und doch realen Carlos, mit dem sie von nun an in eine erst mit ihrem Tod abreißende Abfolge von Figuren der Liebe eintritt.

Medium dieser Liebesfiguren ist in erster Linie die Sprache: Ständige Verdoppelungen und Spiegelungen prägen die Schreibweise Juana Borreros, die ihre Liebesbriefe bisweilen mit ihrem Namen, bisweilen mit „Carlota“ oder „Desdemona“ und häufiger noch mit „Yvone“, der Protagonistin eines romantischen Gedichts des Kolumbianers Abraham Z. López Penha, unterzeichnet. Hinter Carlos Pío Uhrbach, einem Nachfolger Casals in der Ästhetik eines kubanischen modernismo, erscheint immer wieder das Bild des 1893 verstorbenen Dichters; und Juana Borrero wird in ihren Briefen nicht müde zu betonen, sie habe Carlos schon

22 Juana Borrero: Epistolario, Bd. 1, S. 372. 
lange bevor sie ihn kennenlernte geliebt und bewusst gewählt. Diese oftmals in unzähligen Variationen betonte und reflektierte Tatsache der eigenen aktiven Rolle erscheint bisweilen mit drohendem Unterton:

Jetzt, in der Stille meines Zimmers, alleine mit mir selbst, begreife ich, ach!, dass ich Dich mehr liebe, als Du es vermutest! Und zwar schon seit geraumer Zeit! Denn Du weißt sehr wohl, dass ich es in dieser Geschichte war, die die Initiative ergriff, Dich zu lieben. ${ }^{23}$

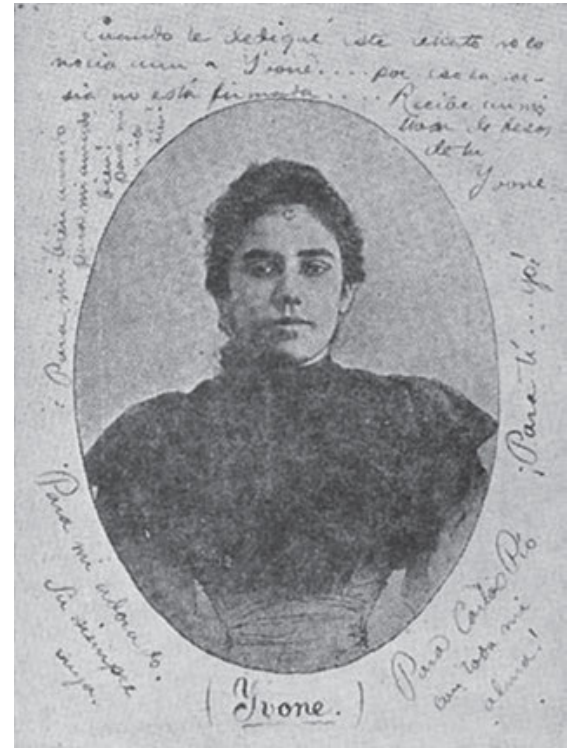

Abb. 104: Porträt von Juana Borrero „Yvone“, auf dem Titelblatt der Rimas, Carlos Pio Uhrbach gewidmet, 1895.

Juana Borrero hat in dieser Beziehung, in dieser „Geschichte“, die Initiative ergriffen und in einer patriarchalischen Gesellschaft den männlichen Part übernommen. Fiktion und Wirklichkeit, Literatur und Leben in Briefform überlagern sich zunehmend, bis sie ununterscheidbar werden. Das Leben entsteht aus den Spiegelungen der Literatur, welche die Silhouetten des Lebens aus den Reflexen einer absolut gestellten Liebeskonzeption in Bewegung setzen. Das Spiel der Schatten an der Wand, die Figuren der Liebe zwischen beiden Liebenden ist von der jungen Frau handgemacht: ein Schattenspiel, aus Händen gebildet.

Das Leben wird, gegenläufig zu Nietzsches Frauenbild, zu einem Traum, der durch Liebe Leben schafft und direkte Kommunikation gerade dadurch herstellt, dass Unmittelbarkeit unterbunden wird:

23 Borrero, Juana: Epistolario, Bd. I, S. 138. 


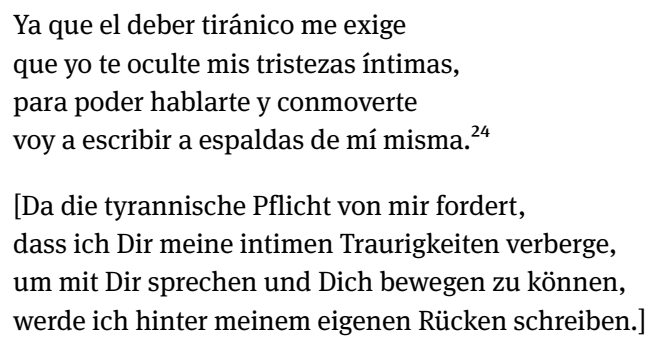

Das Ich durch das Ich umbringen und sich selbst als schöne Leiche bestaunen sowie ein Schreiben hinter dem eigenen Rücken: Wohl nur auf diese Weise lässt sich die Liebe zu einem Mann verstehen, der nicht Mann sein soll und gerade deshalb geliebt wird, weil er mehr als ein Mann ist. Dieser Aufstand der Geschlechter und die Verwirrung der Subjekte schließt dabei stets die Dimensionen von Revolte und Rebellion der jungen Frau innerhalb einer patriarchalischen Gesellschaft ein. In der Verdoppelung der Liebesobjekte spiegelt sich ein Subjekt, dessen modernistische Ästhetik nicht nur die Literatur und das Lesen, sondern auch Liebe und Leben auf grundlegende Weise erfasst.

Die außersprachliche Wirklichkeit wird damit außer Kraft gesetzt, zumindest zeitweise, bis die Familie aufgrund der konspirativen Tätigkeit Esteban Borreros, ${ }^{25}$ der für die Sache Martís eintritt, aus Gründen der persönlichen Sicherheit ins Exil gehen muss und Kuba im Januar 1896 in Richtung Florida verlässt, wo Juana wenige Wochen später den Tod fern ihrer Insel finden wird. Das Leben greift in Form der kollektiven Geschichte ein und macht eine letzte Anstrengung notwendig, die Absolutheit des Liebesanspruches als Fusion von Leben, Lesen und Lieben dramatisch und kunstvoll zugleich vorzuführen. Aber mit welchen Mitteln und auf welche Weise kann dies geschehen?

Die Antwort der Arzttochter Juana Borrero ist so einfach wie medizinisch plausibel: Die Substanz des Lebens selbst wird in Literatur verflüssigt, indem Juana Borrero dem Empfänger in absoluter Radikalität einen nicht mit (einer ,literarischen') Tinte, sondern mit ihrem eigenen (,lebendigen') Blut - „mit dieser Art von Tinte, welche Dir die Hälfte meiner Gedanken einflößen wird“26 _ geschriebenen Brief zu lesen gibt. In diesem blutroten Schreiben stellt sie ihn in grausamer Zuspitzung vor eine Lebens- und Liebesalternative:

24 Borrero, Juana: Ya que el deber titánico me exige ... In (dies.): Poesías. La Habana: Academia de Ciencias de Cuba 1966, S. 106; das Gedicht ist auf den 15. März datiert.

25 Zur Freundschaft Julián del Casals mit seiner ,antítesis viviente“ Esteban Borrero sowie den weiteren Mitgliedern der Familie Borrero vgl. Armas, Emilio de: Casal, S. 116ff., hier S. 117.

26 Borrero, Juana: Epistolario, Bd. 2, S. 256: „en esta clase de tinta que te sugerirá la mitad de mis pensamientos“. 
Oh mein einziges Gut meiner Seele, niemals fühlte ich mich zu Beginn eines Briefes an Dich so traurig. Niemals war die Sprache so ungehorsam und auch so ungenügend. Aus diesem Grunde wollte ich Dir mit dieser Art von Tinte schreiben, welche Dir die Hälfte meiner Gedanken einflößen wird ... Ich habe mir die Venen des linken Armes geöffnet, meines so sehr Dir gehörenden Armes, den ich so vertrauensvoll und zärtlich immer auf Deine Schulter stütze. Dies wird Dir meine absolute Leidenschaftslosigkeit im Angesicht der physischen Qualen beweisen. Mit derselben Indifferenz würde ich es von meiner Stirne oder von meinem Herzen laufen sehen. Überdies will ich, dass Du die Worte dieses Briefes niemals mehr vergisst. Ich spreche zu Dir im Namen von etwas, das Dir mehr wert sein müsste als hundert Vaterländer, von etwas, das genauso heilig ist wie das Vaterland selbst. Ich werde zu Dir von meiner Trauer sprechen. In diesem Brief, den ich mit meinem eigenen Blute schreibe, will ich Dir meine ganze Liebe in einem einzigen klingenden Seufzer einfangen ... in einem einzigen Angstschrei, in einem Klagen und Anklagen ...! Ich will Dir sagen, dass mir das Leben wie eine Last schwerfällt, und wenn ich lebe, so ist es durch und für Dich. [...]

Auf die eine oder andere Art, ist etwa das Vaterland, la Patria, nicht eine Rivalin wie jede andere auch? Und eine glückliche Rivalin, denn Du opferst mich ihr! Es scheint Dir schmachvoll, ihrem Rufe nicht zu folgen ... und es scheint Dir nicht verbrecherisch, mit einem einzigen Schlage alle Hoffnungen einer Seele wie der meinigen zu zerstören? Wenn Dich meine Tränen nicht bewegen, wenn bei Dir die Gewissheit meines Todes nicht den Ausschlag gibt, woraus ist dann Dein Herz gemacht? Entweder Dein Vaterland oder Deine Juana: Wähle Du. Wenn Du gehst, verlierst Du mich. ${ }^{27}$

Dieser Brief ist in der Tat erschütternd! In ihm kommt in der spanischen Formel quiero zugleich die Liebe und der Wille, das „Ich-liebe-Dich“ und das „Ichwill“ in absoluter Zuspitzung zum Ausdruck. Es ist ein Brief mit allem, was ein Körper-Leib zu bieten hat und verzweifelt in die Waagschale werfen kann. Erneut stoßen wir hier auf seine Präsenz im Schreiben auf allen - und diesmal wirklich allen - Ebenen.

Das Schreiben ist ein einziger Liebes- und Angstschrei, ausgeführt mit dem kühlen Schnitt einer erfahrenen Arzttochter, welche das rinnende Blut nicht in Schrecken versetzt. Es ist schon erstaunlich, dass Juana Borrero mit der impasibilidad und der indiferencia jene beiden Begriffe auswählt, die bei Gustave Flaubert mit Blick auf seine Roman- und Sezierkunst zugleich als zentrale Voraussetzungen seines analytischen, kühlen und doch aufwühlenden Schreibens bezeichnet wurden. Die Liebe wird zum eigentlichen Leben, für das die physischen Funktionen völlig sekundär geworden sind. Denn das eigentliche Leben ist die Literatur, die als fundamentale Lebensform und zugleich Lebenspraxis fungiert, während der Lebenssaft umgekehrt in die Literatur - im wahrsten Sinne des Wortes - einfließt. Äußerste Schärfe und äußerste Emotion, welch ein chassé-croisé: Liebe in ihrer extremsten Steigerungsform!

27 Borrero, Juana: Epistolario, Bd. 2, S. 256f. 
Um der Bedrohung des eigenen Lebens durch das bedrohte Leben des Anderen zu entgehen, verflüssigt in diesem blutroten Brief vom 11. Januar 1896 die Literatin kraft ihrer Liebe das eigene Leben in einer äußersten Kraftanstrengung, die das Risiko eingeht, „mit einem einzigen Schlage“ Leben, Liebe und Lesen für immer buchstäblich zu liquidieren. Doch das Leben schrieb die Geschichte anders, denn Juana sollte als erste im Exil sterben, bevor Carlos Pío, der sich doch noch den aufständischen Truppen anschloss, am Weihnachtstag des folgenden Jahres fiel - wie lange vor ihm José Martí, der eigentliche Vater des Befreiungskrieges. Die großen Figuren der kubanischen Lyrik und der kubanischen Intelligenz erlebten Jahrhundertwende und Gründung der kubanischen Republik nicht mehr: ein Aderlass ohnegleichen und von verheerenden Folgen für das gesamte 20. Jahrhundert Kubas. Es gibt ein Schweigen in der kubanischen Geschichte und Kultur, das wir von Beginn des 20. Jahrhunderts an nicht länger überhören sollten. Lassen Sie mich an dieser Stelle etwas emotional hinzufügen, dass dieses Schweigen im Grunde noch heute auf der Insel wie im Exil $\mathrm{zu}$ hören ist!

Mit ihrem Blut wie ihren über dem Brief vergossenen Tränen unterstrich Juana Borrero buchstäblich ihren mehrfach geäußerten Anspruch, José Martí ähnlich, in Gänze, mit ihrem Körper wie ihrem Leib, in ihren Briefen anwesend zu sein. Cintio Vitier hat zutreffend darauf hingewiesen, dass die Verwandlung der handschriftlichen Briefe in ein gedrucktes Buch mit identischen Druckbuchstaben eine mediale „Übertragung“ darstellt, bei der ungeheuer viel verloren gehe - neben der blutroten Schrift nicht zuletzt auch die zahlreichen Tränen, die Flecke auf dem Briefpapier hinterließen. Und der Lyriker, der einst der kubanischen Orígenes-Gruppe um José Lezama Lima angehörte, fügte einfühlsam hinzu:

Diese Schrift, in welcher noch der Puls der Hand und des Herzens zu spüren ist, die sie schrieben, diese so häufig von den Tränen benetzte Schrift, deren Spuren das Papier dunkel färben und die Tinte entfärben, diese fieberhafte, winzige, unwiderstehliche Schrift, welche in einem gewissen Augenblick mit dem Blut der eigenen durchschnittenen Venen und stets mit dem Blut der Seele geschrieben wurde, diese Schrift in die abstrakten Buchstaben der Maschine oder in den Druck zu überführen, kommt der Übertragung aus einer lebendigen Sprache in die Steifheit einer toten Sprache gleich. ${ }^{28}$

Das Körper-Leib-Kunstwerk der Blutschrift Juana Borreros ist folglich intermedial nicht in ein anderes Medium zu übersetzen, da der Körper der kubanischen Dichterin durch diese Übertragung gleichsam herausgefiltert wird. Als erkaltete Herzensschrift nahm die Literatur freilich ihren Körper auf. Das von Juana Borrero

28 Vitier, Cintio: Las cartas de amor de Juana Borrero, S. 8. 
verschriftlichte Herzblut stellt eine nochmalige Radikalisierung eines körperlichen Schreibens mit einer anderen Körperflüssigkeit, jener der Tränen dar, welche bis heute sichtbar das Briefpapier der kubanischen Künstlerin sinnlich punktieren und ästhetisch präsentieren. Eine Grenze wird hier überschritten, die lebensgefährlich ist für den Lesenden wie für die Liebende und (noch) Lebende; wird damit doch ihr Blut aus dem Kreislauf herausgepresst, um in Schrift zu erstarren. Alle Seiten dieser liebenden Schrift der kubanischen Dichterin sind im Grunde Kunstwerke für sich.

Die Liebe der Juana Borrero war fordernd in einem absoluten, das Leben in ein Kunstwerk transformierenden Sinne. Nur vor einem solchen Horizont kann es als geradezu moderat erscheinen, dass Juana ,ihrem ‘ Carlos Monate zuvor das Versprechen abnötigte, ihre rein(e) symbolhafte Liebe niemals körperlich zu vollziehen. Das weibliche Subjekt schützt sich davor, zum weiblichen Objekt zu werden, indem es ,sein‘ Liebesobjekt zugleich vergöttlicht und fest-stellt, für immer in der Unkörperlichkeit des Nicht-Mannes in ihrem eigenen Niemannsland fixiert.

In den ersten Junitagen des Jahres 1895, als José Martí längst im von ihm entfesselten Krieg gestorben war - ob den Heldentod oder den Freitod, war in Abhängigkeit von der jeweiligen ideologischen Position in Kuba seit jeher umstritten -, schickte „Deine keusche Gemahlin“, „Deine süße Braut“, „Deine Yvone“29 Carlos Pío Uhrbach einen Brief, der mit der Gebetsformel „Oh Maria! ¡bendita eres entre todas las mujeres!“(Oh Maria, gebenedeit bist Du unter allen Frauen) ${ }^{30}$ absichtsvoll marienhaft begann. Die religiös-christliche Marien-Anrufung war gut gewählt, wurde im nachfolgenden Brief doch gleichsam ein Ehevertrag (als „ein unersetzliches Dokument*31) bekräftigt und unterzeichnet, das als Eheversprechen den (körperlichen) Vollzug der Ehe auf immer aussetzte. Doch hatte Carlos Pío Uhrbach auch wirklich verstanden, worum es Juana Borrero ging?

Beunruhigt erkundigte sie sich bei ,ihrem‘ Mann, ob er verstanden habe, dadurch auf „die Logik Deiner Rechte“ zu verzichten, um sofort hinzuzufügen: „Ich will, dass Du immer mein Idol bist ...“32. Und triumphierend dann:

(Oh Maria, gebenedeit bist $\mathrm{Du}$

unter allen Frauen!)

Mein vergötterter Carlos,

Soeben habe ich Deinen beruhigenden Brief erhalten. [...] Ich beschwöre Dich beim Andenken Deines Vaters, der für Dich das Heiligste ist, dass Du mich keine Hoffnungen bilden lässt,

29 Borrero, Juana: Epistolario, Bd. I, S. 158.

30 Ebda., S. 155.

31 Ebda.

32 Ebda. 
welche nicht verwirklicht werden. Was Du mir versprichst, ist für mich transzendent, so süß, so groß, so beruhigend, so dass ich es niemals gewagt hatte, dies mehr als nur zu träumen, und als ich Dich kennenlernte, konnte ich auf meine Träume nicht verzichten ... Eben darum, weil ich Dich so sehr liebe! Denke und überlege.

Oh meine Liebe! Wie groß und rein bist DU! Wirst Du es immer sein!? ... Dies ist, was so wichtig ist! Meine Fürbitten sind nicht für jetzt ... Verstehst Du mich? Sie sind nicht für jetzt! Sie sind wenn ... für dann, wenn ich der Logik Deiner Rechte weichen muss ... Verstehst Du mich? ... Für dann ist es! ... Denke und überlege. [...] Du hast Recht! Du und ich, wir sind außergewöhnliche Wesen ... Wir haben die Verbindung zwischen Körper und Seele zerbrochen, wir haben das dumpfe und degradierende Joch der körperlichen Heimsuchungen abgeworfen ... Wir können stolz darauf sein, rein zu sein ... aus einer anderen Erde als die Allgemeinheit gemacht zu sein! ${ }^{33}$

Diese ganze Passage atmet noch immer, atmet von neuem das amabam amare des Augustinus, das uns seit Beginn unserer Vorlesung wohlvertraut ist. Juana Borrero liebt nicht nur ,ihren' Carlos, sondern gerade auch ihre eigene Liebe zu ihm, die sie immer mehr zuspitzt und im Sinne einer radikalen Außerordentlichkeit versteht. Da muss auch der Liebespartner mitmachen! Ob Carlos Pío Uhrbach wirklich versteht, wie radikal der Ansatz seiner Geliebten ist? Sie selbst zweifelt ja daran, wie ihr mehrfaches Nachfragen verrät. Wieder erkennen wir jenes amabam amare, auf das uns Denis de Rougemont im Zusammenhang mit dem Mythos von Tristan und Isolde aufmerksam machte.

Im Ausdruck des Glücks über die ,ideale und himmlische Vermählung zweier Zwillingsseelen, welche sich, ihrer selbst sicher, einander übergeben“"34 war jene ideale Liebesformel gefunden, die nicht nur die sakralisierte Jungfräulichkeit Marias, sondern auch die Literatur der Gemelas (Zwillinge) - jenes Lyrikbandes der Brüder Uhrbach, der Juanas Liebe nicht auslöste, aber ihr eine neue Richtung gab $^{35}$ - in die erträumte Lebenspraxis überführte. Oder wäre diese Jungfräulichkeit, die einen langen dogmengeschichtlichen Streit innerhalb der Kirche auslöste, der erst um die Mitte des 19. Jahrhunderts mit der Verkündung des Dogmas von der unbefleckten Empfängnis Mariens kirchenrechtskräftig ,entschieden` wurde, ein schlichter Übersetzungsfehler? Vieles scheint dafür zu sprechen ... ${ }^{36}$ Doch wollen

33 Ebda.

34 Ebda., S. 157: „nupcia ideal, celeste de dos almas gemelas que se entregan una a otra seguras de sí mismas".

35 Auch im Rückgriff auf den Titel dieses Lyrikbandes erweist sich die absichtsvolle Vernetzung von Lesen, Leben, und Lieben in Juana Borreros Denken und Schreiben.

36 Vgl. hierzu die folgende Stellungnahme von Julia Kristeva zum dogmengeschichtlich so wichtigen Streit: „Il semblerait que l'attribut „vierge“ pour Marie soit une erreur de traduction, le traducteur ayant remplacé le terme sémitique désignant le statut socio-légal d'une jeune fille non mariée, par le terme grec parthenos qui spécifie quant à lui une situation physiologique et psychologique: la virginité. On pourra y déchiffrer la fascination indo-européenne, analysée 
wir uns hier auf die kreativen Kräfte konzentrieren, welche das Dogma der katholischen Kirche bei Juana Borrero und vor allem in ihrem Schreiben auslöste.

Denn diese Selbstbemächtigung des Subjekts, diese weibliche Subjektwerdung lässt zugleich die ästhetische Tragweite jener paradoxen Verschränkung hervortreten, die Leben, Lieben und Lesen in Juana Borreros Lebens-Werk bilden und die am treffendsten vielleicht in ihrem Sonett „Apolo“ zum Ausdruck kommt:

Marmóreo, altivo, refulgente y bello, Corona de su rostro la dulzura, Cayendo en torno de su frente pura En ondulados rizos sus cabellos.

Al enlazar mis brazos a su cuello Y al estrechar su espléndida hermosura Anhelante de dicha y de ventura La blanca frente con mis labios sello.

Contra su pecho inmóvil, apretada Adoré su belleza indiferente, $\mathrm{Y}$ al quererla animar, desesperada,

Llevada por mi amante desvarío, Dejé mil besos de ternura ardiente Allí apagados sobre el mármol frío! ${ }^{37}$

Zweifellos ist dieses Gedicht eine der herausragenden künstlerischen Schöpfungen einer Dichterin, die so früh schon ihren ästhetischen Höhepunkt erreichte. In diesem im Sinne Nietzsches Apoll und nicht Dionysos gewidmeten Sonett, in dem die bildhauerische Plastizität des französischen Parnasse unüberhörbar mitschwingt, wird die Schönheit eines Männerbildes vorgetragen, das in die Regungslosigkeit eines „ídolo“oder - wie sich Juana Borrero in ihrem Tagebuch ausdrückte eines „Ideals des Mannes, den ich erträumt““,38 der als Mann kein Mann mehr wäre, überführt wurde. Auch in diesem Sinne ist ,ihr‘ so schöner Apoll in-different.

par Dumézil, pour la fille vierge comme dépositaire du pouvoir paternel; on peut y voir aussi une conjuration ambivalente, par spiritualisation excessive, de la déesse mère et du matriarcat sous-jacent avec lequel se débattaient la culture grecque et le monothéisme juif. Toujours est-il que la chrétienté occidentale orchestre cette „erreur de traduction“, qu’elle y projette ses propres fantasmes et y produit une des constructions imaginaires les plus puissantes que l'histoire des civilisations ait connues.“ Kristeva, Julia: Histoires d'amour, S. $298 \mathrm{f}$.

37 Borrero, Juana: Apolo. In (dies.): Poesías, S. 80. Auch hier belasse ich es bei der spanischen Originalfassung und überlasse der Leserin und dem Leser die Übersetzung.

38 Borrero, Juana: Epistolario, Bd. I, S. 40. 
Die Umwandlung des Männerbildes in ein Marmorbild, die Verwandlung der erotischen Umarmung in das Versiege(l)n eines Kusses, die chromatische Einbettung einer Regungslosigkeit, welche durch die Betonung von ,indiferente ${ }^{\text {“39 }}$ psychologisiert, aber vergeblich animiert wird, überlässt dem lyrischen Ich jenen Spiel-Raum, in dem die Gesten und mehr noch Figuren der Liebe erprobt und in Szene gesetzt werden können. In dieser Choreographie der Liebe, die das liebende Subjekt (Roland Barthes nach) zugleich konstituiert und aufführt, wird das männliche liebende Subjekt zugleich fest-gestellt und kalt-gestellt, entfaltet die „brennende Zärtlichkeit“ ihr Liebes-Spiel doch auf der plastischen Oberfläche des „mármol frío“, des kalten Marmors. Die romantische Überhitzung der Figuren wird in die distante Chromatik modernistischer Ästhetik übersetzt, ohne doch ,ihr“ Göttliches, ihr „ídolo“ aus dem Blick zu verlieren. Die Kunst gestaltet hier ein Leben, das sich selbst als Kunstwerk inszeniert und gerade darum immer schon Leben ist. Eben diese ästhetische Einsicht in den Zusammenhang von Leben und Kunst, von Leben und Literatur beherrschte die Lebens-, Liebes- und Kunstauffassung der Juana Borrero.

Auch wenn aus dem in unserer Vorlesung gewählten Blickwinkel die Spannbreite modernistischen Schreibens allein in Kuba schon als schier unermesslich erscheint, sollten wir doch nicht vergessen, dass José Martí, Julián del Casal und Juana Borrero ein Absolutheitsanspruch eint, der die Kunst stets in das Leben eingreifen lässt und umgekehrt das Leben als Kunstwerk inszeniert. Auf die Objektivierung der Frau durch den Mann in einer patriarchalisch bestimmten Gesellschaft antwortet höchst bewusst, und zugleich Juana Borreros eigenes Leben zerreißend, die Objektivierung des Mannes durch die Frau.

Doch so verschieden auch immer die modernistischen Visionen ausfallen mochten, stets herrscht höchste Kontrolle über die Bilder, gleichviel, ob es sich um das Bild des aufopferungsbereiten Kämpfers für das Vaterland, ${ }^{40}$ das Bild des filigranen Dekadenten und seiner Chinoiserien bei Casal oder das kreativ und mit letzter Konsequenz angeeignete Bild der „Virgen triste“, der traurigen Jungfrau, handelt. So unterliegt den Versos libres Martís, den Bustos y Rimas Casals und den Rimas Juana Borreros das gleiche ästhetische Streben, die Absolutheit des

39 Die von Casal zitierte Fassung des Gedichts betonte das in Versendstellung hervorgehobene „indiferente“ durch eine Lexemrekurrenz noch zusätzlich, indem sie bereits im ersten Vers ein „indiferente“ enthält, das später (?) durch „refulgente“ ersetzt wurde.

40 Zur Beziehung dieses Bildes zum Lebensprojekt José Martís und der Anfachung des Krieges vgl. Ette, Ottmar: Imagen y poder - poder de la imagen: acerca de la iconografía martiana. In: Ette, Ottmar / Heydenreich, Titus (Hg.): José Martí 1895 / 1995. Literatura - Política - Filosofía Estética. Frankfurt am Main: Vervuert 1994, S. 225-297. 
Anspruchs auf Subjektwerdung in ein selbstgewähltes Lebensprojekt zu transfigurieren, das in der „Reinheit“, der pureza des selbstbestimmten Zieles Baudelaires Diktum vom absoluten Modern-Sein in die zeitgenössischen Kontexte übersetzt. All dem hat sich auch die jeweilige Liebeskonzeption zu- und gegebenenfalls unterzuordnen - bis hin zur Selbstaufgabe.

Stets ist dieses Modern-Sein des Modernismo im kubanischen Kontext aber auch geschlechtlich modelliert. An dieser Stelle wird der gender trouble, der nur zum Teil ein „Unbehagen“ der Geschlechter ist, von größter Wichtigkeit für Juana Borrero, Julián del Casal oder José Martí, ist er doch stets auf bestimmten Ebenen mit Revolte, Rebellion oder Revolution verknüpft. Hören wir zu diesem gender trouble Judith Butler:

To make trouble was, within the reigning discourse of my childhood, something one should never do precisely because that would get one in trouble. The rebellion and its reprimand seemed to be caught up in the same terms, a phenomenon that gave rise to my first critical insight into the subtle ruse of power: The prevailing law threatened one with trouble, even put one in trouble, all to keep one out of trouble. Hence, I concluded that trouble is inevitable and the task, how best to make it, what best way to be in it. ${ }^{41}$

An die Stelle der Beziehung zwischen den Kulturen tritt bei Juana Borrero der Versuch, die Beziehung zwischen den Geschlechtern neu zu regeln, ein Versuch, der in der patriarchalisch bestimmten Familienstruktur im Hause Borrero ebenso scheitern musste wie in der zeitgenössischen kubanischen Gesellschaft, die den Frauen nur einen klar bestimmten Lebens- und Liebesraum einräumte. Lesen war hier der erste und wichtigste Schritt, um sich einen eigenen Raum, A Room of One's Own im Sinne von Virginia Woolf, zu schaffen. Daher die ungeheure Bedeutung der Lektüre, die auch für Juana Borreros Lieben und Liebesbegehren als Grundlage und Ausgangspunkt dienen musste.

Selbst noch ihre Krankheit zum Tode, der sie symbolhaft erst im Exil erlag, war noch das Signum einer Rebellion, die keine männliche Stimme, auch nicht jene Julián del Casals, an die Stelle ihrer eigenen Stimme treten ließ. Und diese Stimme war die einer anderen Moderne, deren Impuls aus dem gender trouble kam. Eine Stimme freilich, die lange Zeit nicht gehört wurde und die hinter der Rede vom ,Wunderkind“ verschwand.

In Juana Borreros Schreiben manifestiert sich mit nicht geringerem Nachdruck als in jenem Martís ein Anspruch auf eigene Teilhabe an der Moderne und am hispanoamerikanischen Modernismo. Borreros geschlechtliche Modellierung

41 Vgl. hierzu Butler, Judith: Gender trouble. Feminism and the Subversion of Identity. New York: Routledge, S. vii. 
verfolgten in der zweiten Hälfte des vergangenen Jahrhunderts Delmira Agustini, Juana de Ibarbourou, Gabriela Mistral, Alfonsina Storni und viele andere so eindrucksvoll weiter, dass der peruanische Marxist und Essayist José Carlos Mariátegui im letzten seiner in Lima erstmals 1928 erschienenen Siete ensayos de interpretación de la realidad peruana hervorheben konnte, dass die Dichterin, die „poetisa“, zu einem „Phänomen unserer Epoche“ - und damit war zweifellos die Moderne gemeint - geworden sei. ${ }^{42}$

Juana Borrero war in diesem Sinne eine Vorläuferin, die ihren verzweifelten Geschlechterkampf als Kampf im Bereich einer Liebeskonzeption führte, welche ganz bewusst Neuland betrat und die alles beherrschende Position des Mannes in Frage stellte. Großes Glück ist Juana dadurch nicht beschieden gewesen, denn sie zahlte dafür unter den familiären und gesellschaftlichen Bedingungen ihrer Zeit einen hohen Preis. Aber in der mit Tristan und Isolde vergleichbaren Tragik einer Liebesauffassung, in welcher das amabam amare des Augustinus so unschwer zu hören war, steckten die Kräfte für eine ungeheure kreative Erweiterung der Ausdrucksformen dessen, was die Gesellschaften im Westen erst in der zweiten Hälfte des 20. Jahrhunderts langsam zu transformieren begann. Darin liegt die politische Dimension von Juana Borreros unbedingter und rebellischer Liebe begründet.

42 Mariátegui, José Carlos: Siete ensayos de interpretación de la realidad peruana. Barcelona: Editorial Crítica 1976, S. 265. 